\title{
DON JUAN ODER DIE LIEBE ZUR HAUSMUSIK WAGNER-KRITIK IN EDUARD MÖRIKES ERZÄHLUNG ,,MOZART AUF DER REISE NACH PRAG““
}

Vorab sind zwei Fragen zu klären. Erstens: warum hat Mörikes Mozart-Novelle überhaupt etwas mit Wagner zu tun? Und zweitens: welchen Erkenntnisgewinn verspricht das Wissen, daß eine der wichtigsten nachromantischen Künstlernovellen Mozart im Titel führt und Wagner betrifft? Der hohe Rang der Mörikeschen Novelle in der Erzählkunst des 19. Jahrhunderts ist von diesem Wissen nicht abhängig; er ist längst unbestritten. Und das Verständnis der Erzählung war bisher keinesfalls so lückenhaft, daß man auf einen Schlüssel für alle Geheimnisse hätte warten müssen, ${ }^{2}$ heiße dieser Schlüssel auch Wagner.

Zur ersten Frage: warum hat die Mozart-Novelle etwas mit Wagner zu tun? Der Mozart der Erzählung ist auf der Reise zur Uraufführung des Don Giovanni in Prag. Bei einer unfreiwilligen Zwischenstation spielt er seinen Gastgebern Stücke aus der neuen Oper vor. Es schließt sich ein Kunstgespräch an, in dessen Verlauf Mozart äußert: , ,Je nun, im Lauf der nächsten sechzig, siebzig Jahre, nachdem ich lang fort bin, wird mancher falsche Prophet aufstehen'". ${ }^{3}$ Man kann nachrechnen: genau 60 Jahre nach dem Don Giovanni, 1847, komponierte Richard Wagner den Lohengrin. Wagner war, das haben die Mörike-Kommentatoren längst festgestellt, der Vornehmste unter den prophezeiten falschen Propheten. Mehr steht zunächst von Wagner nicht in der Novelle.

Den Mörike-Forschern ist allerdings bislang ein philologisches Detail entgangen, das in einem viel umfänglicheren Sinne den Mörikeschen Text mit dem Thema ,,Wagner' in Verbindung bringt. Mörikes Freund David Friedrich Strauß veröffentlichte 1851 die Lebensgeschichte des frühverstorbenen Freundes Christian Märklin, in der bis in Details hinein die Schlüsselszene von Mörikes Novelle vorgebildet ist: die musikalische Soirée nämlich, an die sich die Ahnungen vom frühen Tod der Zentralfigur anschließen. ${ }^{4}$ Private musikalische Aufführungen vor allem Haydnscher, Mozartscher und Beethovenscher Musik, wie sie Strauß im Christian Märklin schildert, gehörten zur häufigen Praxis eines Freundeskreises, ${ }^{5}$ an dem neben Strauß auch Mörike Anteil hatte und der die Wagner-Gegnerschaft gewissermaßen systematisch betrieb, man unterhielt z.B. Verbindungen zu Franz Lachner in München. Diese Wagner-Gegnerschaft blieb nicht wirkungslos. Nietzsche hätte sich sonst schwerlich in seiner ersten ,,Unzeitgemäßen Betrachtung' gerade mit den musikalischen Auffassungen des „Bildungsphilisters” Strauß befaßt und zur großen Genugtuung Richard Wagners so schonungslos mit ihnen abgerechnet. ${ }^{7}$ Mörikes Mozart stammt also aus einer musikalischen Clique, die selbst der Wagnerianer Nietzsche ernst genug nahm, um sie zu verhöhnen.

Noch ein anderer Punkt der Straußschen ,,Quelle' für Mörikes Mozart 
ist interessant. Strauß beschreibt im Christian Märklin das Leben und den frühen Tod eines Mannes, der mit seinem politischen Engagement 1848 ein geradezu prototypisches Scheitern erlebt hat. Das weckt eine Reihe von Assoziationen: in der Mozart-Novelle spielt der Vorabend der Französischen Revolution eine beträchtliche Rolle. Die Abfassungszeit der Novelle fällt in das Tief der nach-achtundvierziger Depression. In den Kontext von 1848 gehören aber auch Richard Wagners Schriften ,,Die Kunst und die Revolution', ,Das Kunstwerk der Zukunft' und ,,Oper und Drama".

Diese flüchtigen Hinweise genügen vielleicht schon, um damit auch die zweite vorbereitende Frage zu beantworten: welchen Erkenntnisgewinn das Thema Wagner-Kritik in Mörikes Mozart-Novelle verspricht. Man kann es in symbolischen Jahreszahlen ausdrücken: durch Mozarts Don Giovanni ist die Epoche von 1789 angesprochen. 1848 ist der Schatten über der Entstehungszeit von Mörikes Novelle. Nietzsches Strauß-Kritik bezieht sich auf die ,Kulturbarbarei” der Gründungsbürger von 1871, denn Mörike war, was Wagner anbelangt, ein Gesinnungsgenosse von Strauß. Mörikes Mozart-Novelle rückt demnach ins Zentrum einer kulturgeschichtlichen Epoche, die mit den - man kann so sagen - großen bürgerlichen Daten von 1789 und 1871 bezeichnet ist. Das heißt natürlich nicht, daß Mörikes Novelle auch nur annähernd zu allen grundsätzlichen Problemen Stellung nimmt, die die Geistesverfassung dieses kulturgeschichtlichen Abschnitts prägen. Vielmehr stellt sich jetzt erst die eigentliche Frage, welches Problem die Erzählung nun tatsächlich aufgreift und wie sie es behandelt. Der Weg zur Antwort ist einfach: ich stelle Mörikes eigenen Mozart zwischen den Mozart von 1787 und den Wagner von 1850.

Zunächst also Mozart: das Finale des Don Giovanni . Der Steinerne Gast ist geladen, die Tafel ist gedeckt, Don Giovanni fordert seine Musikanten auf, mit der Tafelmusik zu beginnen. Er verspricht ihnen ,fürstliche Belohnung" für ihre ,frohen Weisen", , vielleicht weil er weiß, daß es die letzte Tafelmusik in seinem Hause sein wird und er die Belohnung nicht mehr bezahlen muß. Undenkbar ist eine solche Ironie Mozarts nicht, der ja genug Erfahrung mit fürstlichen Hausmusiken hatte.

Zur Musik seiner Hausmusikanten probiert Don Giovanni genießerisch die Speisen. Man weiß von einem früheren Fest im Hause Don Giovanni, daß der Herr kein Hungerleider ist. Um Zerlina zu verführen, hatte er damals sogar die Bauern eingeladen zu ,Schokolade, Kaffee, Wein und Speisen, ' zu ,Gefrornem” und ,Konfekt' . ${ }^{8}$ Heute, für den Steinernen Gast, gibt es ,Fasan”, vielleicht nur als präliminarischen Gang, und ,exzellenten Marzimino". 9 Don Giovanni genießt. Mehr noch als die Speisen allerdings genießt er den Anblick seines Dieners. Leporello muß seinen Herrn mit ,Appetit" essen sehen und dabei ,Hunger' leiden. Wenn auch Leporello nicht der Mann ist, vor Hunger, wie er sagt, zu sterben. Leporello verzehrt seine Portion heimlich, und wieder genießt es Don Giovanni, den Mundräuber Leporello bloßzustellen. Schließlich eini- 
gen sich Herr und Diener darauf, daß alles die Schuld des Koches sei: ,,Ja, mein Koch sucht seinesgleichen/Ihm kann keiner widerstehn' ${ }^{10}$

Den Sinn dieser ersten Szene des Finales - gleich nach dem Preis des Koches tritt Donna Elvira auf, Don Giovanni ein letztes Mal vor seinem Verderben zu warnen - den Sinn dieser ersten Szene hat Mozart an einer Stelle musikalisch besonders stark verdichtet. Don Giovanni sieht den Neid Leporellos und steigert dessen Pein durch den herrischen Befehl „Teller”! Leporello gehorcht mit einem „zu dienen“, und gerade jetzt beginnen die Haus- und Hofmusikanten Don Giovannis ein neues Stück zu spielen. Sie spielen die Arie des Figaro aus Mozarts eigener Oper Figaros Hochzeit

\footnotetext{
Nun vergiß leises Flehn, süßes Kosen

Und das Flattern von Rose zu Rosen;

Du wirst nicht mehr die Herzen erobern, Ein Adonis, ein kleiner Narziß. ${ }^{11}$
}

Das klingt wie eine Art musikalischer Solidarität der musizierenden mit den servierenden Dienern. Der Herr möge nur Musik, Teller und Frauen befehlen. Wenn die Diener gehorchen, wissen sie doch, daß das Ende längst nahe herbeigekommen ist: ,,du wirst nicht mehr die Herzen erobern'.

Das Figaro-Zitat lautet allerdings nicht nur zuungunsten Don Giovannis, es richtet sich auch gegen Leporello. Was ist Leporello im Vergleich mit Figaro für ein kläglicher Diener! Zu Beginn der Oper singt Leporello noch ,Ich will selbst den Herren machen/Mag nicht länger Diener sein', ${ }^{12}$ um dann auf eine einzige Drohgebährde Don Giovannis hin für den Rest der Oper klein beizugeben: ,,ich will nichts, ich bin schon still'. ${ }^{13}$ Figaros Herausforderung an seinen Herrn, ,Will der Herr Graf den Tanz mit mir wagen" ${ }^{14}$ hätte Leporello nie riskiert. Und so spielen Don Giovannis Hausmusikanten auch nicht diese eigentliche Erkennungsarie Figaros, sondern Figaros Spott auf das Milchgesicht Cherubino. Figaros Hohn „,Cherubino, auf zum Siege/Auf zum hohen Waffenruhm'" ${ }^{15}$ ist auch Hohn auf die Niederlagen Leporellos.

Das Figaro-Zitat im Don Giovanni ist ganz unmißverständlich auf das Thema ,Herr-Diener' gemünzt. Dieser Gegenstand gab ja dem Mozartschen Figaro die Brisanz. In Beaumarchais' Le mariage de Figaro waren die strittigen Punkte gewiß aggressiver formuliert. Doch auch Mozarts Publikum, bis hinauf zum Kaiser, wußte, was gemeint war.

Erstaunlich sind die Verschiebungen, die das Herr-Diener-Thema dann kurz darauf im Don Giovanni erfahren hat, den nur zwei Jahre vom Beginn der Französischen Revolution trennen. Der Adlige, der in Figaros Hochzeit nur den Verführer spielt, ist jetzt ein Mörder, ein Verführer und ein verstockter Verbrecher dazu. Die Diener, die in Figaros Hochzeit auf eher spielerische Weise ihren Herrn überlisten und blamieren, sind jetzt geprügelte Untertanen und mißbrauchte Handlanger. Am Schluß von Figaros Hochzeit folgt der vollendeten Blamage des Grafen eine schnelle Versöh- 
nung aller. Der verstockte Don Giovanni hingegen endet mit einer Höllenfahrt und die Übriggebliebenen feiern ihre moralische Überlegenheit: „Also stirbt, wer Böses tat". ${ }^{17}$ Es ist im Don Giovanni alles erbarmungsloser geworden. Die eher märchenhafte Einkleidung des drama giocoso täuscht nicht darüber hinweg, daß es nur noch Untergang oder Überleben, keine Versöhnung mehr gibt.

Man kann noch einen Schritt weitergehen in der Deutung des Mozartschen Selbstzitats, der Figaro-Arie im Don Giovanni. Mozart arrangiert das Potpourri der Hausmusikanten Don Giovannis gewissermaßen operngeschichtlich. Das Figaro-Stück ist eingerahmt von Fragmenten aus Martin y Solers Cosa rara und Sartis Fra $i$ due Litiganti. Es ist bekannt, daß Fra i due Litiganti eine der Lieblingsopern der Wiener war, und daß Cosa rara die Beliebtheit des Figaro beim Wiener Publikum direkt beeinträchtigte. Nach der Ironie auf Don Giovanni und auf Leporello steckt in der Kombination der drei Opern-Zitate auch eine Selbstironie Mozarts. Und wie die Figaro-Arie Kommentar auf den Herrn Giovanni und auf den Diener Leporello war, so ist sie zuletzt auch Kommentar auf den Opernkomponisten Mozart. Seine Musik, seine Opern werden zusammen mit denen seiner Kollegen gespielt zu einem Fest, das sich ein adliger Herr über Diener und Musikanten zu seinem eigenen Untergang bereitet. Seine, des Opernkomponisten Mozart Musik ist Tafelmusik zum Untergang. Figaros Hochzeit und Don Giovanni sind Festmusik zum Untergang des ancien régime. Kürzer und treffender als mit einem einzigen Selbstzitat könnte Mozart sein eigenes Selbstverständnis nicht ausdrücken. Seine Musik ist Dienerin des ancien régime, verharrt daher in dessen Formen. Sie sehnt sich aus dieser dienenden Rolle heraus und hat darum ein geschärftes Bewußtsein für alle Anzeichen des nahenden Untergangs.

Tafelmusik zum Untergang: Nichts anderes hat Mörikes Mozart-Novelle zum Gegenstand. Man muß nur wieder ein paar Verschiebungen armerken, die den ganzen veränderten Horizont andeuten. Es spielen nicht mehr Domestiken, sondern ein gräfliches Haus und ein wahlverwandter Mozart spielen gewissermaßen von gleich zu gleich. Sie spielen Figaros Hochzeit und Don Giovanni wie jede bürgerliche Familie auch als „Hausmusik', für gewöhnlich muß der Klavierauszug den lebendigen Mozart ersetzen. Die Gesellschaft der Novelle spielt die Hausmusik nicht mehr als Begleitmusik zum Untergang einer Epoche, sondern zu den Todesahnungen eines Genies.Man kann diese Verschiebung schematisieren und sagen: die repräsentative Tafelmusik des feudalen Hausstandes ist zur intimen Hausmusik geworden, sie wurde ,,privatisiert' ${ }^{19}$ Entsprechend ist der zur Allegorie auf das Epochenende gesteigerte Untergang Don Giovannis ,privatisiert“ zur Todesmelancholie eines Individuums. Es ist nur konsequent in dieser Verschiebungsreihe, daß an die Stelle des feudalen Kavaliers das geniale Individuum getreten ist.

Was hat nun die private Hausmusik des Mörikeschen Mozart noch zu tun mit der epochal gemeinten Tafelmusik zum Untergang Don Giovan- 
nis? Das ,,erregende Handlungsmoment" in Mörikes Erzählung ist ein kleiner Sündenfall. ${ }^{19}$ Die Mozarts haben auf der Reise nach Prag Station gemacht, Mozart geht im Park des naheliegenden Landschlosses spazieren. Plätscherndes Brunnenwasser und ein Pomeranzenbaum wecken in Mozart die ,Anschauung des Südens", ${ }^{20}$ wecken musikalische Erinnerungen, schläfern Mozarts Bewußtsein ein. Schließlich reicht er unwillkürlich nach einer der Apfelsinen und pflückt sie ab. ,.. . . ja, so weit geht die künstlerische Geistabwesenheit" - und dies sei ausführlicher zitiert, weil es auf die Einzelheiten ankommt -, ,,daß er, die duftige Frucht beständig unter der Nase hin und her wirbelnd und bald den Anfang, bald die Mitte einer Weise unhörbar zwischen den Lippen bewegend, zuletzt instinktmäBig ein emailliertes Etui aus der Seitentasche des Rocks hervorbringt, ein kleines Messer mit silbernem Heft daraus nimmt und die gelbe kugelige Masse von oben nach unten langsam durchschneidet. Es mochte ihn dabei entfernt ein dunkles Durstgefühl geleitet haben, jedoch begnügten sich die angeregten Sinne mit Einatmung des köstlichen Geruchs. Er starrt minutenlang die beiden innern Flächen an, fügt sie sachte wieder zusammen, ganz sachte, trennt und vereinigt sie wieder.'"21

Schon der Anblick zerschnittener Zitrusfrüchte regt bekanntlich die Speichelsekretion an. Mörike beschreibt den einfachen physiologischen Vorgang, daß eine bestimmte Sinneswahrnehmung unvermittelt eine Körperreaktion hervorruft. Mörike veranschaulicht die körperlich-sinnliche Qualität der Sinneswahrnehmung. Dabei ist hier die „Speichelsekretion" eine denkbar unangemessene Bezeichnung, denn sie verfehlt das was Mörike mit der Schilderung des silbernen Messers aus dem emaillierten Etui andeutet, die Sublimierung nämlich der Körperreaktion zum kultivierten Genuß. Die Orange weckt das Durstgefühl, doch wer silberne Obstmesser bei sich führt, begnügt seine ,, angeregten Sinne'" mit der ,,Einatmung des köstlichen Geruchs'. Mozarts Sündenfall ist eine Regung sublimierter Sinnlichkeit, ist ein Akt kultivierten Genusses. Und Musik leitet diesen kultivierten Genuß ein: eine ,,musikalische Reminiszenz" 22 betäubte Mozart, so daß er ,,zerstreut" und ,,zuletzt instinktmäBig" den Frevel am Pomeranzenbaum verübt. Während er die ,,duftige Frucht'" genießt, bewegt er ,,bald den Anfang, bald die Mitte einer Weise unhörbar zwischen den Lippen“. Später erfahren wir, was das für eine Weise war. Es war das Tanzlied von Masetto und Zerlina aus dem Don Giovanni, ,Duett und Chor einer ländlichen Hochzeit'. ${ }^{23}$ Wir erfahren, daß Mozart diese noch fehlende Nummer zu seiner neuen Oper komponiert hat, während in seinem Kopf die musikalische Reminiszenz umging, und während er sich dem sublimierten Orangengenuß hingab. Die musikalische Reminiszenz und das neu komponierte Tanzliedchen machen dabei einen interessanten Kontrast. In seiner Reminiszenz erinnert sich Mozart einer höchst raffinierten musikalischen Darbietung beim König beider Sizilien, einem aufwendig inszenierten Balett im Golf von Neapel. ${ }^{24}$ Die Erinnerung an diese höfische, allerdings auch heitere Prunkmusik ausge- 
rechnet gibt Mozart die Melodie ein, die die Einfachheit, die idyllische Unschuld einer Dorfhochzeit charakterisieren soll. Doch der Kontrast stimmt. Es ist der dramatische Kontrast der Oper Don Giovanni selbst: das höfische Festgebahren Don Giovannis, seine großartigen Verführungsinszenierungen, seine unmoralischen Anschläge auf die Unschuld des Bauernmädchens, in deren ländliche Sittsamkeit und Schlichtheit der höfische Sittenstrolch einbricht.

Noch einmal, beide musikalischen Regungen Mozarts, die höfische Erinnerung und die ländlich-idyllische Komposition, sind verbunden mit dem einfachen, aber kultivierten und sublimierten Genuß der Pomeranze. Es scheint, als sei der kultivierte und sublimierte Genuß hier das tertium comparationis für höfischen Prunk und ländliche Einfachheit.

Mörike enthüllt selbst die Logik, mit der er die Mozartschen Erinnerungs- und Sinnesregungen arrangiert hat. Er läßt Mozart nicht irgendeine Pomeranze pflücken und zerschneiden. Er läßt die Frucht vom Baum einer historischen Erkenntnis stammen. Bei einem „Feste in Trianon”, dem Erdmittelpunkt aller höfischen Kultur, empfing eine Verwandte der Mozartschen Gastgeber ,,den blühenden Orangenzweig" aus der Hand der Madame de Sévigné. Und der Orangenzweig wuchs heran ,,als lebendes Symbol der feingeistigen Reize eines beinahe vergötterten Zeitalters . . ., worin wir heutzutage freilich des wahrhaft Preisenswerten wenig finden können, und das schon eine unheilvolle Zukunft in sich trug, deren welterschütternder Eintritt dem Zeitpunkt unserer harmlosen Erzählung bereits nicht mehr ferne lag. " 25 Bemerkenswert sind die folgenden Größenverhältnisse: „die feingeistigen Reize eines beinahe vergötterten Zeitalters" einerseits, der kurze Moment des kultivierten Apfelsinengenusses andererseits. Der ,welterschütternde Eintritt" einer Zukunft einerseits, die ,harmlose Erzählung“ andererseits. Das paßt zu einer früheren Gegenüberstellung: der Untergang einer Epoche in der Höllenfahrt des Don Giovanni einerseits, die Todesahnungen eines einsamen Mozart andererseits. Die Privatisierung, die Verkleinerung des Epochalen zum Genrehaften hat bei Mörike Methode. Die Privatisierung und die Verkleinerung sind rückübersetzbar, das Genrehafte bei Mörike vergißt seine epochalen Bedingungen nicht. Mörike arrangiert Mozarts Erinnerung an die höfische Musik, die Komposition des ländlichen Liedes und die fast emblematische Deutung des Pomeranzenbaumes so, daß die kultivierte Sinnlichkeit des ancien régime zum Oberbegriff wird sowohl für das höfische Prunkfest als auch für die ländliche Idylle. Das Arrangement der höfischen und ländlichen Kontraste ist historisch sehr aussagekräftig. In Trianon hielt man beide Seiten des Kontrastes für vereinbar. ${ }^{26}$ Marie Antoinette spielte die Schäferin in ihrem künstlichen Dorf mit echten Schafen. Das Ländliche war die Hauptattraktion ihrer Feste in Versailles. Im Don Giovanni wird der Kontrast zum Konflikt. Der Kavalier stört die Idylle, die Bäurin soll mißbraucht werden, der Bauer wird verprügelt, und es endet alles mit dem Strafgericht für den Leuteschinder. Auch Mörike wußte, daß Marie An- 
toinette nicht im Stroh der Schäferin gestorben ist, daß der Genuß des Don Giovanni seine brutale Seite hat. Mozarts ,,vergöttertes Zeitalter' der kultivierten Sinnlichkeit drängt auch bei Mörike auf seine ,unheilvolle Zukunft' hin.

Das alles steckt in dem zur Genre-Szene verkleinerten Sündenfall, dem erregenden Handlungsmoment der Erzählung. Dem Sünder naht der Rächer in Gestalt eines Gärtners, der ihm erst das Ausmaß seines Vergehens vor Augen führt. Die Früchte des Baums waren gezählt, sie sollten bei der bevorstehenden Verlobungsfeier der Tochter des Hauses einzeln besungen werden. Weil es ursprünglich neun Pomeranzen waren, handelte das Gedicht natürlich von den Musen, und denen fehlt nun eine. Mozart wird vom Gärtner dingfest gemacht, von dessen Herrschaft freudig erkannt und mit seiner Frau zum Verlobungsfest eingeladen. Es wird musiziert, es wird ein ,,ausgedehnter Schmaus“ gehalten, es wird erzählt, sehr viel erzählt, es wird auf dem „Gipfel geselliger Lust" 27 getanzt und es wird geschenkt. Die Mozarts schenken dem Brautpaar ein hölzernes Salzfaß, das mit Hilfe seiner ausführlichen Herkunftsgeschichte erhoben wird zum „Muster patriarchalischer Simplizität”..28

Was sich auf dem Landschloß während des Mozartschen Besuches abspielt, entspricht ganz dem Lebensstil eben eines Land-Schlosses: die patriarchalische Simplizität und die gesellige Lust, die aristokratischen Tafelfreuden und das bürgerliche Hausrat-Schenken. Es ist das idyllische Zerlina-Masetto-Motiv, die Simplizität der ländlichen Hochzeit, übertragen in die kultivierte Atmosphäre eines Hauses aus dem ancien régime. Inbegriff der kultivierten Simplizität aber, Gipfel des ästhetischen Genusses, den die Schloßgesellschaft sich selber bereitet, ist die Hausmusik: ,,Eines hatte den Flügel geöffnet, ,Figaros Hochzeit' lag aufgeschlagen, und das Fräulein schickte sich an, von dem Baron akkompagniert, die Arie Susannas in jener Gartenszene zu singen, wo wir den Geist der süßen Leidenschaft stromweise, wie die gewürzte sommerliche Abendluft, einatmen. Die feine Röte auf Eugeniens Wangen wich zwei Atemzüge lang der äußersten Blässe; doch mit dem ersten Ton, der klangvoll über ihre Lippen kam, fiel ihr jede beklemmende Fessel vom Busen. Sie hielt sich lächelnd, sicher auf der hohen Woge, und das Gefühl dieses Moments, des einzigen in seiner Art vielleicht für alle Tage ihres Lebens, begeisterte sie billig." 29

„,Süße Leidenschaft', , ,gewürzte sommerliche Abendluft', ,,das Gefühl dieses Moments, des einzigen seiner Art für alle Tage ihres Lebens": es sind die Elemente einer hochgradig sublimierten Sinnlichkeit, die sich in dieser Art von Hausmusik steigern zum höchsten Lebensgefühl. Und für Mörike liegt in einer solchermaßen, genossenen' Hausmusik das beste Erbe aus der Kultur des ancien régime.

Man kann die Bedeutung der Mörikeschen Hausmusik erst ganz ermessen, wenn man diesen Inbegriff der kultivierten Genuß-Atmosphäre aus dem ancien régime vergleicht mit dem, was bei anderen Leuten, bei 
Zeitgenossen Mörikes „Hausmusik' heißt.

Wilhelm Heinrich Riehl, der konservative Sachwalter der bürgerlichen und der deutschen Familie, verkündete die Ideologie der ,,deutschen Hausmusik': , . . . sie ist uns Urkunde für den Geist der . . . bürgerlichen, nicht der vornehmen Kreise'. Die ,,schlichte, ehrliche deutsche Hausmusik' ist ,,rein und züchtig', und sie ist das genaue Gegenteil einer ,,mark- und knochenlosen" Musik, in der das ,,blasirte, kranke, zerrissene, überweibliche Wesen der feinen Welt"' sein ,,erschreckend wahres Spiegelbild”' findet. ,,Blasiertheit, Frivolität, Sentimentalität, Geckerei und Zügellosigkeit" machen die mark- und knochenlose Musik zum „,Fluch des Hauses'. ${ }^{30}$,,Kranke'" Salonmusik und ,,gesunde' Hausmusik - man weiß, wie zählebig diese hygienisch-ästhetische Zweiteilung der Welt geblieben ist.

Riehl hätte sich geschüttelt, wenn er gerade die kultivierte Sinnlichkeit des ancien régime, die sublimierte Frivolität wirklich ,,vornehmer Kreise“ zum Lebenselement der Hausmusik hätte erheben sollen. Und Riehls Weltbild wäre ins Wanken gekommen, wenn er die Teilung in ,vornehm“ und ,,bürgerlich“, in ,,krank“ und ,gesund“ hätte für die Idee der Hausmusik aufgeben müssen. Mörike macht ja gerade die Hausmusik seines Mozart-Kreises zum Ort der Aufhebungen, zum Ort der Integration von ,vornehm“ und „einfach“, von „feudalem Genuß” und „,bürgerlicher Tugend". Das höfische Ballett und die schlichte Dorfmusik hatten im Moment der kultivierten Sinnlichkeit den Punkt ihrer Koinzidenz. In Mörikes Konzept der Hausmusik steckt das Idealbild einer Menschlichkeit, die im kultivierten Genuß Trennungen überwindet und so zum ,höchsten' Lebensgefühl befähigt. Mörike hat sein Ideal mit dem Idealbild des ancien régime verbunden, seine Sehnsüchte waren gewiß rückwärts gewandt. Doch er wußte, daß sein ancien régime ein sehr poetisches Idealbild war, nicht anders als das Mittelalter des Novalis. Er hätte sonst nicht so deutlich von der unheilvollen Zukunft gesprochen, von der die Wirklichkeit des ancien régime betroffen war: Ludwig XVI. und Mozart starben im selben Jahr 1791.

Riehl sah nichts Vereinigendes im Genuß der Hausmusik, es sei denn für die häuslichen Familienmitglieder. Genuß war gar nicht zulässig, und trennen, scharftrennen vom Kranken sollte den Bürger die Hausmusik.

Auch Riehl entwarf ein Idealbild, sogar ein Zukunftsbild vom Bürgerhaus im 20. Jahrhundert: das wird so schön, daß sich sogar die ,Engel . . . freuen über solch ein Haus, und musiciren dazu mit ihrer himmlischen Hausmusik.' ${ }^{31}$ Im Gegensatz zu Mörike hat Riehl nichts von der , unheilvollen Zukunft' seiner Wunschbilder gewußt.

Wenn man Mörikes und Riehls Begriffe der Hausmusik einander gegenüberstellt, weiß man, warum Adorno die Kammermusik das „Korrektiv des patzigen Bürgers“" genannt hat. Der ,,patzige Bürger“, „der auf dem Seinigen steht”, ganz wie Riehl, wird korrigiert durch die „Courtoisie“, durch die ,soziale Tugend der Höflichkeit”. Adorno meinte das Aufein- 
anderhören-Können der Kammermusik-Stimmen. Er meinte die Tatsache, daß das Ganze der Kammermusik sich nicht konstituiert ,durch die auftrumpfende Selbstbehauptung der einzelnen Stimmen - sie ergäbe ein barbarisches Chaos". ${ }^{32}$ Mörike betonte ein anderes Korrektiv des Barbarischen, die kultivierte Sinnlichkeit. Ob „Höflichkeit” der Kammermusik oder „Kultiviertheit” des Hausmusik-Genusses - beides sind, wie wieder Adorno es nennt, ,umfunktionierte Erinnerung(en) an feudale Elemente, die dem Gang des Fortschritts zum Opfer fielen". Solche Wiederkehr des zu unrecht Vergessenen - das wäre die geeignetste Formel für Mörikes Genuß der Musik im Hause des ancien régime. ${ }^{33}$

„Fortschritt” lautete die letzte Reizvokabel. Ein Fortschritt, gegen den sich trotz aller sonstigen Unterschiede Mörike und Riehl ${ }^{34}$ einig wissen konnten, ein revolutionärer Fortschritt in der Musik, der nicht nur die musikalischen Ideen eines Mörike und Riehl vergessen machte: Richard Wagner. In der Zeit, in der Mörike seine Mozart-Novelle verfaßte, ist es der Wagner des Tannhäuser und des Lohengrin, der Wagner, der mit diesen beiden Opern von fast allen deutschen Opernbühnen gespielt wurde (, auch von den Hoftheatern, obwohl Wagner im Exil lebte' ). ${ }^{35}$ Es ist der Wagner der Schriften Die Kunst und die Revolution, Das Kunstwerk der Zukunft, Oper und Drama, deren ,intellektuelle Herausforderung" ${ }^{\prime 36}$ mit dem Erfolg von Tannhäuser und Lohengrin zusammentraf. Und es ist der Wafner von der Schopenhauerlektüre. Es kann hier nicht alles angesprochen werden, was für den Wagner der 1850er Jahre „Fortschritt” bedeutete. Man müßte da zum Beispiel auf die Rolle Feuerbachs eingehen. Man käme auf dem Umweg über diese philosophische Lektüre Wagners unweigerlich auf das Thema,,Sinnlichkeit”. Aber auch über eigene Stichworte Wagners, die die hier angesprochenen Gegenstände betreffen, gelangt man ins Zentrum der Wagnerschen Gedankengänge. Ein solches Stichwort - das Wort bei Wagner in seiner pluralischen Bedeutung genommen - gibt Wagner anläßlich des Don Giovanni:

„Wenn . . . vor der ersten französischen Revolution unter einer ganzen Gattung frivolgenußsüchtiger Menschen die Stimmung vorhanden war, in der ein Don Juan die allerbegreiflichste Erscheinung, den wahren Ausdruck dieser Stimmung ausmacht . . . so war die Wirkung einer solchen Darstellung zu jener Zeit gewiß eine ganz bestimmte und unzweifelhafte auf das Gefühl. Wie steht es nun aber, wenn heute, vor dem gänzlich veränderten, börsengeschäftlichen oder geheimregierungsräthlichen Publikum der Gegenwart, und von einem Darsteller, der gern Kegel schiebt und Bier trinkt, und dadurch aller Verführung entgeht seiner Frau untreu zu werden . . .? Wird dieser Don Juan nicht mindestens ganz anders verstanden, als es die Absicht des Dichters war, und ist dieses ganz andere... nicht in Wahrheit gar kein Verständnis des Don Juan mehr?"37

Die Feststellung, daß die Philister den Don Juan nicht mehr begreifen können, will Wagner freilich nicht mißverstanden wissen als Aufforderung, die Philister sollten zur ,frivolgenußsüchtigen” Gattung vor der 
ersten französischen Revolution zurückkehren. Die Söhne der zweiten französischen, der Julirevolution hatten ja ein viel besseres ,Evangelium der Sinnlichkeit', sie verkündeten eine revolutionäre, fortschrittliche „Emanzipation des Fleisches”. Die ,,gesunde Sinnlichkeit" 38 sollte doch Ausdruck gerade einer Freiheit werden, an der alle Menschen Teil haben können. Sie sollte gerade die Verhältnisse beseitigen, in denen die Vielen Not leiden, damit die Wenigen genießen können. Verhältnisse sollte diese Freiheit beseitigen, die die Existenzgrundlage jener ,,Gattung frivolgenußsüchtiger Menschen" des ancien régime und des restaurierten ancien régime waren. ${ }^{39}$

,Das Volk - schreibt Wagner - ist der Inbegriff aller Derjenigen, welche eine gemeinschaftliche Noth empfinden." Das Volk kennt nur wirkliche, zur Not gesteigerte Bedürfnisse. Das Volk kennt nur die Natur des Leibes als die Grundlage der Sinnlichkeit, den ,,wirkliche(n), sinnliche(n) Hunger”. Den „Luxus“ der Reichen und Privilegierten, der ,,ebenso herzlos, unmenschlich, unersättlich und egoistisch“ ist wie „das Bedürfnis, das ihn hervorruft", diesen Luxus, der die ,,Seele (der) Industrie'" und , ,die Seele unseres Staates ist' , ihn wird die echte Sinnlichkeit des Volkes, die Not hinwegfegen. ,,Die Noth wird die Hölle des Luxus endigen; sie wird die zermarterten, bedürfnislosen Geister, die diese Hölle in sich schließt, das einfache, schlichte Bedürfnis des rein menschlich sinnlichen Hungers und Durstes lehren . . . gemeinsam werden wir wirklich genieBen, gemeinsam wahre Menschen sein. Gemeinsam werden wir aber auch den Bund der heiligen Nothwendigkeit schließen, und der Bruderkuß, der diesen Bund besiegelt, wird das gemeinsame Kunstwerk der Zukunft sein" 40

Das ist deutlich genug: die Sinnlichkeit, zu deren Genuß einer statt Durst zu spüren sich nur eine mit silbernem Messer zerlegte Orange unter die Nase hält, solche ,,bedürfnißlose' Sinnlichkeit belegt Wagner mit einem harschen Luxusverbot. Zur Tugend wird buchstäblich die Not erklärt, nur in der Not sind wir natürliche Menschen, weil wir nur in Hunger und Durst unsere Natur ganz spüren. Das ist ein auffallendes Argument bei eimen Revolutionär, der gegen den um den Preis des Industrie-Sklaventums erkauften Luxus die Befreiung einer liebenden Menschheit von der ,Sorge" ${ }^{41}$ setzt. Verkehrt sich angesichts der Realitäten der Industriegesellschaft die Anstiftung zur Sorglosigkeit in vorbeugende Verherrlichung der Not? Deutet Wagner an, daß die Beseitigung des Luxus noch nicht die Beseitigung des Sklaventums heißt? Wie soll das Angebot einer freien Sinnlichkeit glaubwürdig werden, wenn Entsagung und Entbehrung zu ihren Vor-Bedingungen erklärt werden? Wie auch immer: Das Gemeinschaftssymbol des in der Entbehrung natürlichen und sinnlichen Volkes, das Wahrzeichen der sich erhebenden Not-Gemeinschaft ist das ,,Kunstwerk der Zukunft”' sprich, das Wagnersche „,Drama".

Mozarts Don Giovanni umschrieb selbst den historischen Typ der Oper, 
den er verkörperte. Er bezeichnete sich selbst als ,,Tafelmusik des untergehenden ancien régime'". Wagners Tannhäuser umschreibt seinen eigenen historischen Charakter nicht minder deutlich: er handelt vom Sängerkrieg. Die Wagnersche Oper ist gerade dort, wo sie das Musikalische selbst thematisiert, ganz auf Kampf eingestellt. Die Meistersinger streiten mit der Musik um Rückschritt oder Fortschritt. Der Don Giovanni betrachtet wie der Figaro das Schicksal der Herrschenden aus der Perspektive der Diener, so wie der Komponist zu den musizierenden Dienern gehörte. Wagner stellt den Kampf dar, er betrachtet ihn trotz aller Identifikationen von außen. Denn er ist der freie, ,freischwebende" Künstler geworden, der zwar nach Maßgabe seiner eigenen Bedürftigkeit Partei ergreift für die Bedürftigen, der aber ohne Schaden an seiner Identität zu nehmen auch anders Partei ergreifen kann. Wagner hat zuletzt immer genug Distanz zu den kämpfenden Parteien, um ihren Kampf als Gesamtschauspiel ,,von oben" thematisieren und gestalten zu können. Mozart dagegen sprach bis hin zur Zauberflöte immer mit der Moral einer der kämpfenden Parteien. Doch zurück zu den speziellen Fragen.

Tannhäuser hätte durchaus das Zeug dazu, ein ganzer Don Juan zu werden. Nach seinen Erlebnissen mit Frau Venus tut er sich ja schwer, die Erwartungen des Tugendapostels Wolfram und des landgräflichen Hofes gerecht zu werden. Und der Verrat an Elisabeths treuer, engelreiner Liebe hat durchaus etwas von Don Giovannis Verrat an der immerhin unverbrüchlichen Liebe Donna Elviras. Doch das sind nur Oberflächenentsprechungen. Schon im Venusberg selbst herrscht eine dem Don Giovanni ganz und gar fremde Sinnenlust. In der Bühnenanweisung zum Bacchanal des Tannhäuser heißt es: ,,Von Neuem belebt sich der Tanz und gelangt zu dem äußersten Grade wilden Ungestüms. - Mit dem Momente der trunkensten bacchantischen Wut tritt eine schnell um sich greifende Erschlaffung ein. $"{ }_{42}$ Don Giovanni gerät weder in Wut, noch erschlafft er. Don Giovanni lacht allenfalls, und bis zu allerletzt verfährt er nach seiner Maxime: ,Mag die Welt in Trümmer gehn,/Niemand soll mich zagen sehn!'43

Wie eine Replik auf diesen Trotz Don Giovannis klingt Tannhäusers Vorsatz ,Zerknirschung sei mir Lust“ ${ }^{44}$ Tannhäuser wird allerdings rückfällig mit seiner ,unheimlichen Lüsternheit“. ${ }^{45}$ Um ihn darum endgültig vor der „Hölle Lust“ ${ }^{46}$ zu bewahren, wird ihm der Liebes- und Erlösungstod zuteil: erlöst wird er von seinem Leib, dem Ursprung aller bösen Sinnlichkeit. Wo die Not die Bedingung der Sinnlichkeit ist, wird nur der Tod, das Ende aller Not, zu ihrer Befriedigung. Die Befriedigung ist nichts anderes als Erlösung von der Not der Sinnlichkeit. Auch am Tannhäuser bewahrheitet sich, was für Wagners Auffassung von der Sinnlichkeit schon oft bemerkt worden ist. Wagnersche Sinnlichkeit unterliegt den schärfsten Normen des von Wagner scheinbar so verachteten ,,geheimstaatsräthlichen" Publikums. Auch Wagner kennt die Entladung der Sinnlichkeit nur als "Wut", als eine Wut, die in der "Erschlaffung” 
die Quittung für ihre Unbotmäßigkeit bekommt.

Hier zeigt sich die gewissermaßen stillschweigende Evidenz der Mörikeschen Wagner-Kritik. Einen Mozart aus dem ,frivolgenußsüchtigen", ancien régime mit allen Attributen einer raffinierten und kultivierten Sinnlichkeit, einen solchen Mozart nebenbei von Wagner als einem falschen Propheten sprechen zu lassen, das war Wagner-Kritik, die ins Zentrum traf. Sie besagt nicht mehr und nicht weniger, als daß gegenüber der kultivierten Sinnlichkeit der Privilegierten vor der ersten Französischen Revolution die auf Not und Reue gegründete Sinnlichkeit des Wagnerschen Volkes nicht Fortschritt bedeutet, sondern Fortschritt predigt und Verarmung bringt.

So steht es natürlich bei Mörike nicht annähernd. Und es so platt und direkt zu formulieren heißt auch, die ästhetische Methode der Mörikeschen Kritik ganz und gar mißachten. Wieder ist bei Wagner selbst ein Stichwort zu finden, das helfen kann, Mörikes ästhetisches Verfahren zu verdeutlichen.

Von Wagner wird die Äußerung berichtet, ,,er höre zuweilen bei Mozart im Geist das Klappern des Geschirrs zur Tafelmusik." 47 Wir wissen inzwischen, wie Recht Wagner mit seiner Wahrnehmung hat. Was Wagner vielleicht nicht ganz ohne Überheblichkeit gegenüber dem berühmtesten der musizierenden Diener des ancien régime sagte, das umschreibt exakt dessen eigene Selbsteinschätzung: Tafelmusik zum Untergang. Oder: das Klappern des Geschirrs und der ,,welthistorische Moment" gehen im Don Giovanni eine ästhetisch tragfähige Verbindung ein. Das äußerlich Banale verhilft dem menschheitsgeschichtlichen Sinn erst zu seiner besonderen ästhetischen Gestalt. Und dieser ästhetische Reiz des Banalen ist genau der Punkt, an dem Wagners Bemerkung über das Mozartsche Geschirrklappern sich gegen Wagner selbst richtet. Tellerklappern wäre für den ästhetischen Effekt Wagnerscher Opern absolut tödlich. Oder, wie Nietzsche es formuliert: „Wie prüft man (den) ewigen Gehalt" der Wagnerschen Texte? ,Man übersetzt Wagner in's Reale, in's Moderne - seien wir noch grausamer! in's Bürgerliche . . . Nichts unterhaltender, nichts für Spaziergänge mehr zu empfehlen ... zum Parsifal als Candidaten der Theologie mit Gymnasialbildung (-letztere als unentbehrlich zur reinen Thorheit) " ${ }^{48}$

Man kann diese Probe bekanntlich leicht nachmachen, auch auf den Wagner, den die Mörike-Novelle meint. Man vergegenwärtige sich aus dem Tannhäuser Elisabeths berühmtes ,Dich, teure Halle, grüß ich wieder,/froh grüß ich dich, geliebter Raum . . ."49 Man vergegenwärtige sich, wie Elisabeth den Tannhäuser in dieser Halle der Wartburg empfängt:, ,So stehet auf!/Nicht sollet hier Ihr knien, denn diese Halle/Ist Euer Königreich ..." ${ }^{50}$ Schließlich denke man daran, daß diese Halle der Schauplatz für einen Himmel und Hölle bewegenden Sängerkrieg wird. Und dann nenne man das alles Tannhäusers ,Hausmusik”. Ein wenig ernüchternd wirkt auch hier auf den großen Wagnerschen Stil-Gestus das banale Unter- 
bieten.

Dabei hat diese Probe auf Wagners ,großen Stil" nicht irgendein beliebiges Beispiel zum Gegenstand. Der Spott auf die Tannhäuser-Halle und auf den Sängerkrieg, der sich in ihr abspielt, trifft Wagners Selbstverständnis. Der einsame Sänger im Widerstreit mit der in kämpfende Parteien zerteilten Welt - es war ja schon kurz die Rede davon - ist die Grundfigur der Wagnersche Selbstdarstellung. Im Lohengrin etwa wird der Streit zu einem Rechtsstreit vor Gott und Kaiser, Lohengrin selbst von Wagner stilisiert zum ,einsamen Künstler”, ${ }^{51}$ der in diesem Streit schlichten will. Dieser im buchstäblichen Sinn hoch-stilisierten Selbstauffassung des Künstlers Wagner setzt Mörike einen zum Hausmusikanten verkleinerten Mozart entgegen. Mozart erscheint als Held einer Genre-Szene, als Akteur in einer Biedermeier-Handlung. Der Don Giovanni ist verlegt in die intimen Räume eines Landschlosses. Gewiß ein wirksamer Kontrast zur Großartigkeit der Wagnerschen Helden und Schauplätze, zur Staffage des ,,hohen Stils“.

Es ist vielleicht deutlich, wo die eigentliche Stärke der Mörikeschen Wagner-Kritik liegt. Sie liegt im Unausgesprochenen. Es ist eine ganz andere Mentalität der Polemik als man sie gerade aus der unabsehbaren Fülle polemischer Sätze bei Wagner kennt. Mörike gibt nur durch einen einzigen Satz die polemische Richtung seiner Novelle zu erkennen. Mörike hält seine Genre-Szene, sein Konzept der Hausmusik Wagner einfach entgegen. Und aus dieser Perspektive der ,,Verkleinerung" schon gerät Wagner in eine ungünstige Position. Man weiß, daß so keinesfalls die ganze Wahrheit über Wagner entdeckt werden kann. Doch als Verfahren der Wagner-Kritik, als Methode der Wagner-Bekämpfung ist dieser Kunstgriff der Verkleinerung höchst wirksam. Bei Mörike wurde er vielleicht zum ersten Mal angewandt. Und bei Mörike ist die Verkleinerung, die Übersetzung ins Genre nicht billiger Witz, sondern steht selbst für eine ästhetische Position, die ihr Recht behaupten will.

Gegenüber Mozart bedeutete Mörikes Verkleinerung und Privatisierung keine Entstellung. Die epochale Dimension der ,Tafelmusik zum Untergang' ging in der ,,Hausmusik" nicht verloren. Mörike bekräftigte vielmehr ihren aktuellen Sinn und übertrug in sein Konzept der Hausmusik die zu unrecht vergessene, die verlernte Genußfähigkeit des ancien régime. Die in die Hausmusik gerettete Genußfähigkeit Don Juans war gewiß das Gegenteil von Wagners Empfehlung zur Sinnlichkeit in Not und Entbehrung. Der zur - im übertagenen Sinne gesprochen - petite perception sublimierte Genuß der Mörikeschen Hausmusik wird schließlich gerade durch die Geringfügigkeit seiner Reiz-Impulse gefährlich für Wagner: was Mörike mit der Verkleinerung des Hausmusik-Mozart an Wagner beging, das hat über dreißig Jahre später der Wagner-Renegat Nietzsche mit seiner Übersetzung ,,in's Bürgerliche"'52 fortgesetzt. Es ist angemerkt worden, daß der Wagnersche Stilgestus auf die ,ZZerstörung des Biedermeiers' ziele. Daß er protestiere gegen alles ,,kleinbürgerlich-musikalische Glück im Winkel ... gegen das genrehaft Abgesetzte, bequem 
Überblickbare', ${ }^{53}$ Es sei dahingestellt, ob das eine zutreffende Charakterisierung des Biedermeiers ist. Eines scheint immerhin deutlich zu sein: die sublimierten Sinneswahrnehmungen eines vielleicht biedermeierlichen Autors, sein Stil der kleinen Genre-Szenen haben gegenüber den Zerstörungsgelüsten des „,Großen Stils” durchaus Widerstandskräfte.

\section{Tübingen}

GERHART VON GRAEVENITZ

\section{Anmerkungen}

1. Geringfügig überarbeitete und um einen Anmerkungsteil erweiterte Fassung eines Vortrags, den ich im Februar 1980 in der Universität Amsterdam gehalten habe.

2. ,.. . die ,Meisternovelle" Mozart hat eh und je eine rege Beschäftigung der Kritik ausgelöst." Victor G. Doerksen, ,Die Mörike-Literatur seit 1950. Literaturbericht und Bibliographie," DVjs, 47 (1973), Sonderheft. S.343-397; 368. Zur Auseinandersetzung mit der "Musik" in der Novelle verweise ich auf die Studien von Raymond Immerwahr.

3. Eduard Mörike, Sämtliche Werke in zwei Bänden, hrgb. v. Jost Perfahl, mit einem Nachwort von Benno v. Wiese, Anmerkungen, Zeittafel und Bibliographie von Helga Unger (München o.J.), I, 619. Mörikes Werke werden im folgenden zitiert mit römischer Band- und arabischer Seitenzahl.

4. David Friedrich Strauß, Christian Märklin. Ein Lebens- und Charakterbild aus der Gegenwart (1851), S. 197. Egmonts Todesmonolog hat für Märklin ähnliche Funktion wie für den Mörikeschen Mozart der ,ganze lange, entsetzensvolle Dialog " des Untergangs von Don Giovanni (I, 616f). Die ,Tränen der Zuhörerinnen” (Märklin, S.197.) spielen auch in der Mozart-Novelle eine wichtige Rolle, vgl. insbesondere den Schluß. Ein weiteres Indiz für die Bekanntschaft Mörikes mit dem Märklin bezieht sich auf das Stuttgarter Hutzelmännlein, an dem Mörike 1851 - im Jahr, in dem der Märklin erscheint - die Arbeit wieder aufnimmt: Die Bewandtnis, die es mit dem ,,Klötzle Blei glei bei Blaubeure" (I, 497) hat, wird im Märklin, S. 146, erläutert. Strauß zitiert hier ein eigenes Tagebuch, in dessen Orginal (heute Sammlung Fritz Kauffmann) H. U. Simon vom Marbacher Schiller-Nationalmuseum die „Klötzle“Stelle identifiziert hat. Nach Auskunft von H. U. Simon findet sich in Mörikes Briefen von 1851/52, auch in den Briefen an Mörike, kein Hinweis auf den Märklin. Dennoch scheint es mir mehr als ein Zufall zu sein, daß sich zwei Werke, an denen Mörike zum Zeitpunkt des Erscheinens des Märklin arbeitet, mit dieser Biographie berühren. H. U. Simon danke ich für seine Auskünfte.

5. Mittelpunkt dieses Kreises war der auch im Christian Märklin genannte (S. 197) Ernst Friedrich Kauffmann, einer der ältesten Freunde Mörikes (Vgl. Eduard Mörike. Katalog zur Gedenkausstellung zum 100. Todestag im Schiller-Nationalmuseum Marbach a.N., 1975, bes. S. $104 \mathrm{f}$ ). Mörike schildert in einem Brief vom 20. 3. 1843 die Wirkung des Don Juan-Finales auf ihn: es sang David Friedrich Strauß' (!) Frau, Agnes Strauß-Schebest, begleitet von Kauffmann. Dieser Brief (vgl. I, 1056), gilt im allgemeinen als ,,biographischer Beleg “ für die Mozart-Novelle. - Ganz außer Betracht bleibt hier der sehr enge Klassizismus, der Mörike und seine Freunde unfähig machte, Wagners Leistung anzuerkennen.

6. Zum Wagner-Hintergrund von Nietzsches erster Unzeitgemäßer Betrachtung vgl. Ernst Jäckh, ,Friedrich Nietzsche und David Friedrich Strauß. Beiträge zur ,modernen Kultur' '”, Patria (1909), 210-247.

7. Vgl. Wolfgang Amadeus Mozart, Don Giovanni, Reclams Universalbibliothek 2646, S. 73. Mit Rücksicht auf die Vortragssituation habe ich die deutsche Übersetzung des da Ponteschen Textes benützt, ungeachtet der bekannten Mängel, die dieses Verfahren hat. Zu diesen Mängeln gehört auch, daß der von Wilhelm Zeltner besorgte Text nicht ganz den zu Mörikes Zeit geläufigen Fassungen (Gugler/Wolzogen) entspricht.

8. Don Giovanni, S. 30, S.40.

9. Ebd.. S. 74.

10. Ebd., S. $74 \mathrm{f}$.

11. Wolfgang Amadeus Mozart, Die Hochzeit des Figaro, Reclams Universalbibliothek 2655, S. 27.

12. Don Giovanni, S. 19.

13. Ebd., S. 22. 
14. Die Hochzeit des Figaro, S. 15.

15. Ebd., S. 27.

16. Don Giovanni, S. 80.

17. Auf Cosa rara geht auch Mörikes Novelle ein. Vgl. I,577. Zur Tatsache, daß das musikalische Selbstzitat bei Mozart sogar autobiographische Funktion haben kann, vgl. Zofia Lissa, ,Ästhetische Funktionen des musikalischen Zitats," Musikforschung, 19 (1966), 364 $378,371$.

18. In folgender Passage kommt die ,Privatisierung“ am deutlichsten zum Ausdruck: ,Die Wirkung eines solchen Vortrags in einem kleinen Kreis wie der gegenwärtige unterscheidet sich natürlicherweise von jedem ähnlichen an einem öffentlichen Orte durch die unendliche Befriedigung, die in der unmittelbaren Berührung mit der Person des Künstlers und seinem Genius innerhalb der häuslichen bekannten Wände liegt." (I,586)

19. ,Hier sitze ich Unseliger in Ihrem Paradiese, wie weiland Adam, nachdem er den Apfel gekostet" (I,581). Die Funktion der ,,kleinen Sündenfälle” in Mörikes Werk - so zentral wie hier für die Handlung der Mozart-Novelle - habe ich versucht zu zeigen in Eduard Mörike: Die Kunst der Sünde.Zur Geschichte des literarischen Individuums (Tübingen, 1978).

20. I, 579

21. I, $579 \mathrm{f}$.

22. I, 579.

23. I, 592 .

24. I, $588 \mathrm{ff}$.

25. I. 596.

26. Auf diesen Kontrast hin interpretierbar ist auch die ,,eigentümliche naive Opposition“, die die erste Besitzerin des Orangenzweiges zur Versailler Gesellschaft macht: ,Bei ihrer unbefangenen Teilnahme an jenem steten Wechsel des geistreichsten Lebensgenusses verleugnete sie auf keinerlei Art ... die angestammte deutsche Ehrenfestigkeit und sittliche Strenge ..." (I, 595).

27. I, 601.

28. Die ,Angelegenheit des armen Liebespaares" (I,609), die zur Geschichte dieses Salzfasses gehört, zeigt Mozart gewissermaßen in der Anti-Rolle zu Don Juan. Während Don Juan die Hochzeit Zerlina-Masetto stört, stiftet Mozart eine solche ,idyllische" Verbindung.

29. I, 585.

30. Wilhelm Heinrich Riehl, Die Familie, 9., vermehrte Auflage (1882), S. 210; ders., Hausmusik. Fünfzig Lieder deutscher Dichter in Musik gesetzt, 2., umgearbeitete Auflage (1860) (1. Auflage 1855), ,Des Tonsetzers Geleitbrief', S. XVII, XIX, XXI. Hier, S. XV u. XXII, schon die Gegenerschaft zu Wagner als Motiv der Hausmusik angesprochen.

31. Riehl, Die Familie, S. 303.

32. Theodor W. Adorno, Einleitung in die Musiksoziologie. Zwölf theoretische Vorlesungen, Gesammelte Schriften, Bd. 14, S. 169-433, S. 273.

33. Natürlich hat die Mörike-Forschung das Zeit-Kolorit der Mozart-Novelle nicht unbeachtet gelassen. Aber es erscheint meist trivialisiert zum ,Rokoko" und spielt dann eine ähnliche Rolle wie das Klischee vom ,,verspielten Mozart”. Den ,Rokoko“-Genuß in einem genaueren Sinne auf das Thema ,Sinnlichkeit” im 19. Jahrhundert zu beziehen, wurde unterlassen.

34. Riehl nannte sich ,,einen der ältesten Gegner Wagners”. W. H. Riehl, ,Richard Wagner', in ders., Kulturgeschichtliche Charakterköpfe (1891), S. 443-528, S. 447.

35. Carl Dahlhaus, Richard Wagners Musikdramen (1971), S. 9.

36. Ebd.

37. Richard Wagner, Eine Mittheilung an meine Freunde, Gesammelte Schriften und Dichtungen, Bd. IV, 285-418, S. 300f. (Leipzig 1872).

38. ,Feuerbach's Wort von der ,gesunden Sinnlichkeit' - das klang in den dreissiger und vierziger Jahren Wagnern gleich vielen Deutschen - sie nannten sich die jungen Deutschen wie das Wort der Erlösung." Friedrich Nietzsche, ,,Nietzsche contra Wagner. Aktenstücke eines Psychologen". Nietzsche. Werke, hrgb. v. Giorgio Colli und Mazzino Montinari, Sechste Abteilung, 3. Band, S. 411-437, S. 429.

39. Vgl. die Gegenüberstellung von ,,sorgenlose(m) Reichtum', , ,Lust zum feineren Genusses dieses Reichtums" bei den Fürsten des ancien régime und einer von der "Sorge" befreiten ,,brüderliche(n) Menschheit", die zu ,Liebe”, ,,Schönheit" und ,,Kunst" findet. Die Kunst und die Revolution, Gesammelte Schriften, III, 9-50; 23, 41f. Ähnlich Wagners Selbstdeutung des Tannhäuser: Einerseits ,,Sinnlichkeit und Lebensgenuß . . . in der Gestalt Dessen ..., was unsere moderne Welt als Sinnlichkeit und Lebensgenuß bietet". Andererseits ,,wirkliche Liebe”. Mittheilung an meine Freunde, Ges. Schriften, IV, 342 f. Während 
Nietzsche in ,Richard Wagner in Bayreuth" Wagners Gegenüberstellung - von Scheinbedürfnis/Luxus und Not - noch zustimmend referiert, schreibt er später vom Wagnerschen „,Raffinement als Ausdruck des verarmten Lebens”. „In seiner (Wagners) Kunst ist auf die verführerischste Art gemischt, was heute alle Welt am nöthigsten hat, - die drei grossen Stimulantia der Erschöpften, das Brutale, das Künstliche und das Unschuldige (Idiotische)." Nietzsche, Werke, IV/1, S. 47-49; VI/3, S. 17, S. 41.

Vgl. in diesem Zusammenhang auch Dieter Jähnig, „Die Kunst in der Zeit der Arbeit“ (Nietzsches „Bayreuth-Gedanke”), in ders., Welt-geschichte: Kunst-geschichte. Zum Verhältnis von Vergangenheitserkenntnis und Veränderung (Köln 1975), S. 161-196.

40. Richard Wagner, Das Kunstwerk der Zukunft, Gesammelte Schriften, III, 51-210; 59, $61 \mathrm{f}$.

41. Vgl. Anm. 39.

42. Richard Wagner, Die Musikdramen. Mit einem Vorwort von Joachim Kaiser (1978), S. 223.

43. Don Giovanni, S. 50

44. Wagner, Die Musikdramen, S. 243.

45. Ebd., S. 247.

46. Ebd., S. 250.

47. Theodor W. Adorno, Versuch über Wagner, Gesammelte Schriften, Bd. 13, Die musikalischen Monographien: W'agner, Mahler, Berg, S. 7-148, S. 46.

48. Nietzsche, Werke, VI/3, S. 28. Zu diesem Spott Nietzsches vgl. Adorno, Versuch über Wagner, S. 121.

49. Wagner, Die Musikdramen, S. 232.

50. Ebd., S. 233.

51. Vgl. Mittheilung an meine Freunde,Ges. Schriften, IV, S. 369.

52. Nietzsche setzt auch den Inhalt von Mörikes Wagner-Kritik fort: ,,Selbst Mozart's Verhältniss zur Musik - Wagner hat es uns zum Trost gesagt! - war im Grunde frivol ... Lassen wir niemals zu, dass die Musik ,zur Erholung diene'; dass sie ,erheitere'; dass sie ,Vergnügen mache'. Machen wir nie Vergnügen! - wir sind verloren, wenn man von der Kunst wieder hedonistisch denkt . . . Das ist schlechtes achtzehntes Jahrhundert . . . Nichts dagegen dürfte räthlicher sein, bei Seite gesagt, als eine Dosis - Muckerthum, sit venia verbo. Das giebt Würde." Nietzsche, Werke, VI/3, S. 20.

53. Th. W. Adorno, Versuch über Wagner, S. 61, S. 39. ,.. . . selbst Nietzsche hat Wagner noch mit den Ohren des Biedermeier gehört, als er ihn formlos fand." Ebd., S. $39 f$. 